\title{
Extinction curves of GRB environment with the X-shooter
}

\author{
Andreja Gomboc ${ }^{1}$, Jure Japelj ${ }^{1}$ and Stefano Covino ${ }^{2}$ \\ ${ }^{1}$ Faculty of Mathematics and Physics, University of Ljubljana, \\ Jadranska 19, 1000 Ljubljana, Slovenia \\ email: andreja.gomboc@fmf .uni-lj.si \\ ${ }^{2}$ INAF/Osservatorio Astronomico di Brera, \\ via Bianchi 46, 23807, Merate (LC), Italy
}

\begin{abstract}
Gamma Ray Bursts (GRBs) can be used as a powerful tool to study galactic environments at different epochs of the Universe's evolution, thanks to their bright afterglow emission ranging from X-rays to optical and radio wavebands. Important aspect of the environment is dust, which plays a central role in the astrophysical processes of interstellar medium and in the formation of stars. GRBs can be a unique probe of dust at cosmological distances, where its origin and properties are still poorly known. By using a sample of GRB afterglow spectra observed with the VLT/X-shooter spectrograph we studied the rest-frame extinction in GRB lines-of-sight by modelling the broadband near-infrared to X-ray afterglow spectral energy distributions. We present our results on the rest-frame extinction of our sample, and illustrate that the spectroscopic data, thanks to a combination of excellent resolution and coverage of the blue part of the spectral energy distributions, are more successful than photometric measurements in constraining the extinction curves and therefore the dust properties in GRB hosts.
\end{abstract}

Keywords. galaxies: ISM, dust, extinction, gamma rays: bursts

\section{Introduction}

GRBs are unique and powerful tools to study the origin and properties of dust at cosmological distances. For example, the attenuation of light as a function of wavelength, or extinction curve, is strongly dependent on the composition and size of dust grains. The afterglow emission of GRBs is of a synchrotron nature and has a simple power-law or a broken power-law dependence on frequency. GRB afterglows are therefore better suited for studying the extinction than objects with more complex spectra, such as quasars. Modelling of afterglow spectral energy distribution (SED), from NIR/optical to X-ray regime, provides us with information regarding the intrinsic afterglow spectrum and properties of the material in the line-of-sight (LOS). As dust properties in random LOS in high-redshift galaxies are not a-priori known, representative average extinction curves are usually adopted, i.e. Milky way, Large Magellanic Cloud, and Small Magellanic Cloud average extinction curves. There have been several studies modelling the GRB afterglow SEDs, some using only NIR-UV photometric data, while others extending this to X-ray spectral range (e.g. Covino et al. (2013) analysed SEDs of the BAT6 complete sample of GRBs), and some used also afterglow spectra (e.g. Zafar et al. (2011)).

\section{Modelling SEDs with the $\mathrm{X}$-shooter data}

In our work we took the next step in SED modelling and used spectra obtained with the X-shooter spectrograph at the Very Large Telescope. In the period from 2009 to $2014 \sim 60$ afterglow spectra were obtained as part of the X-shooter GRB GTO program, 
but our final sample consists of only 9 GRBs, 8 long and 1 short (for details on our sample selection see Japelj et al. (2015)).

In SED modelling we used X-shooter and photometric data, as well as the Swift/XRT data to remove the degeneracy between the rest frame extinction $A_{\mathrm{V}}$ and spectral slope $\beta$. We performed 3 types of SED modelling, using: (i) X-shooter data only, (ii) X-shooter and X-ray data, and (iii) photometric and X-ray data. In case (i) we find that using only the X-shooter data usually gives bad fit (good for only $3 \mathrm{GRBs}$ ). In case (ii) we find that $A_{\mathrm{V}}$ falls in the range between 0 and 1.2 , which are values commonly found in GRB LOS. We find that in most cases (6) Small Magellanic Cloud type extinction curve is preferred, in 2 cases Large Magellanic Cloud type, and in 1 case we could not single out the best extinction model, because it has very low extinction. In case (iii) the derived values for $A_{\mathrm{V}}$ and $\beta$ are similar as in (ii), however photometric SEDs can usually be modelled by more than one extinction curve and in most cases we can not strongly favour one over the other.

We also derive $A_{\mathrm{V}}$ and equivalent hydrogen column density as functions of GRB redshifts (for more details see Japelj et al. (2015).

We showed that the spectroscopic data, especially with the high-resolution and excellent coverage in blue part of the SED, can help us constrain dust properties much better than the photometric measurements. However, independent of the quality (resolution) of the NIR-UV data, the SED modelling is secure only when the fit is performed on a broader SED.

\section{References}

Covino, S., Melandri, A., \& Salvaterra, R., et al. 2013, MNRAS, 432, 1231

Japelj, J., Covino, S., \& Gomboc, A., et al. 2015, A\&A, 579, A74

Zafar, T., Watson, D., \& Fynbo, J. P. U., et al. 2011, A\&AA, 532, A143 